Article

\title{
Regularized Reconstruction of HBIM for Built Heritage-Case Study with Chinese Ancient Architecture
}

\author{
QUE Raner ${ }^{1}$, WANG Xi ${ }^{1, *}$, WU Cong ${ }^{1}$, and BAI Chenjun ${ }^{1}$ \\ 1 Room 300, School of Architecture, Tianjin University, NO.92 Weijin Road, Nankai District, Tianjin, China; \\ 444605654@qq.com, wangxi88love@tju.edu.cn, wucong@ju.edu.cn, babybcj@sina.com \\ * Correspondence: wangxi88love@tju.edu.cn
}

\begin{abstract}
By the study of the pattern book Ying Zao Fa Shi (building regulations of Song Dynasty, $1103 \mathrm{AD}$ ), while analyzing the combining and dimensioning rule of timber framework and tile work, a model self-generating program has been compiled for the first time. The operating framework has been firstly defined, while solving the issues of clustering principle, connecting method, output classification, etc. with the detailed description of algorithm theory. Taking the corner bracket set and nine-ridge roof for example, after the compilation and debug by Grasshopper, according to various input parameters, various models have been generated automatically by the plugin, proving the velocity and the veracity of the algorithm.
\end{abstract}

Keywords: Chinese ancient architecture; bracket set; tile work; regularized reconstruction; parametric; algorithm modeling; Grasshopper; HBIM; built heritage

\section{Introduction}

During the restoration and conservation process of Chinese ancitent architecture, the "ontology" (material level) of ancient architecture and the "record" (text level) of design and construction may continuously cause the increase or decrease of the content. With the accumulation of years, the "authenticity" of ancient architecture will discounted with the lack of both material and text level[1].

2D drawing and 3D model failed to express the connecting logic, which is already defined in the pattern book and vital for Chinese ancient architecture[2]. The significance lies in the parametrical connection among each component, of which size, position and form could be translated with equations.

The pattern book Ying Zao Fa Shi firstly been published in 1103 AD concluded the combination and measurement rule of Chinese architecture, along with the standard terms and figures[2]. This book aims to express the method of design, processing and construction by simple rules, with the unique format, just met the idea for computer coding. 

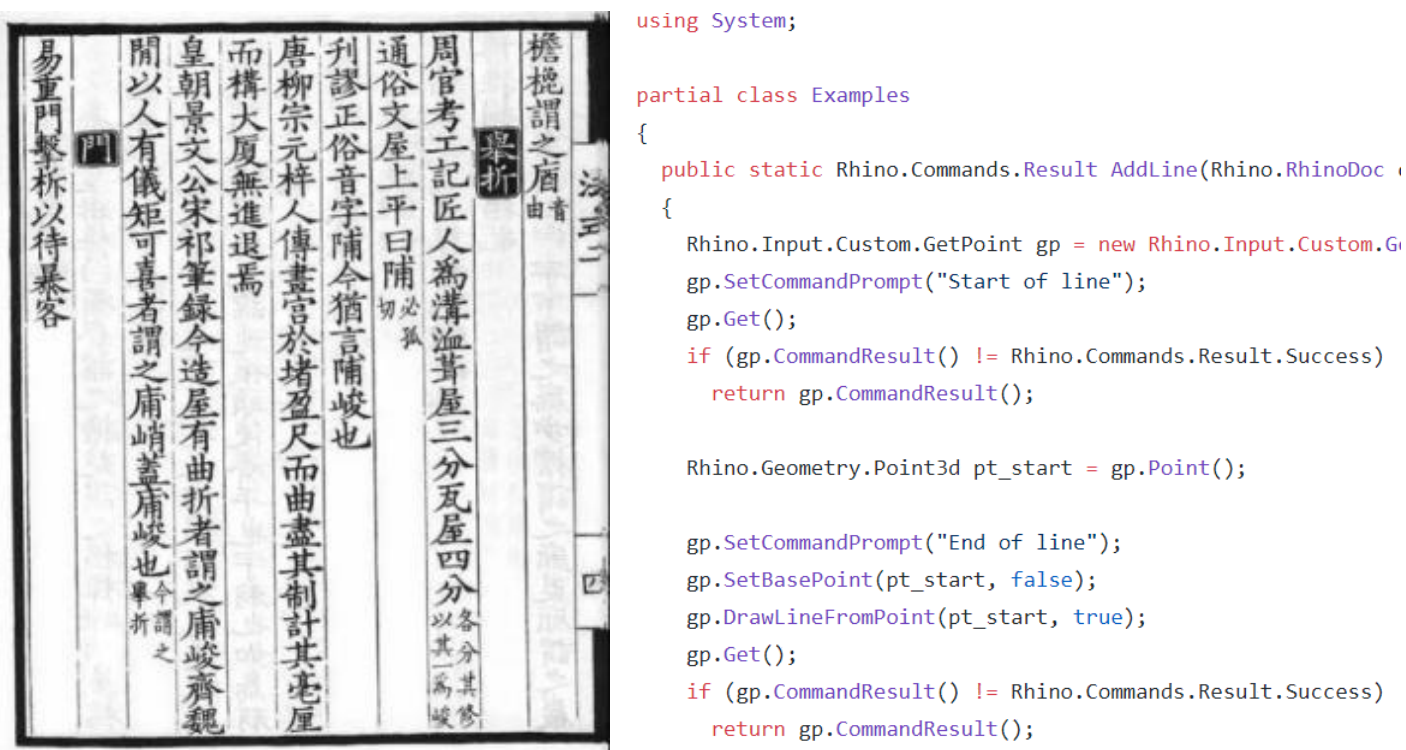

Figure 1. Ying Zao Fa Shi (left) and code format (right)

As is shown in the Figure 1, Chinese ancient architecture includes 4 CLASS by utility class; 13 METHOD by processing method and material. FUNCTION with black shading represent component, SYNTAX without shading represent explanation and example, and the NOTES written by small word just like the notes for codes.

Grasshopper is a node-visible, graphical programing plugin for Rhino[3]. With the NURBS-modeling and data-processing function battery, algorithm modeling would present the full-scale real-time illustration and precise logic, running automatically without manual intervention[3].

\section{Methods}

\subsection{Compiling Principle}

Define a module for calculating numbers then exporting numbers as a Calculator; define a module for processing geometry then exporting geometry as a Generator; define a module with pre-loads data then offering value selections for the user as Selector.

The Selector offers check-down list and ture or faluse $(1$ or 0$)$ switch, based on the requirement of pattern book.

Each module clustered by certain amount of batteries could be clustering to another new module once again.

The measurement would be the Caifencun Unit System. 1 cai stands for a standard wood block, which is 15 fens high and 10 fens wide. The standard component is defined by fen (design size), fen can be transferred into cun (actual size). The actual size of 1 fen alters depends on which grade has been used. Only 1 to 5 grades of cai can be used for Bracket Set. However, 6 grade of cai also be used widely for other part, so some component keeps using 6 grade cai without changing. Normally, 1 cun equals $31 \mathrm{~mm}[2]$.

Therefore, both the accuracy and authenticity of the record and model could be accommodated while compiling with cun and modeling with millimeter. 
2.2 Timber Framework

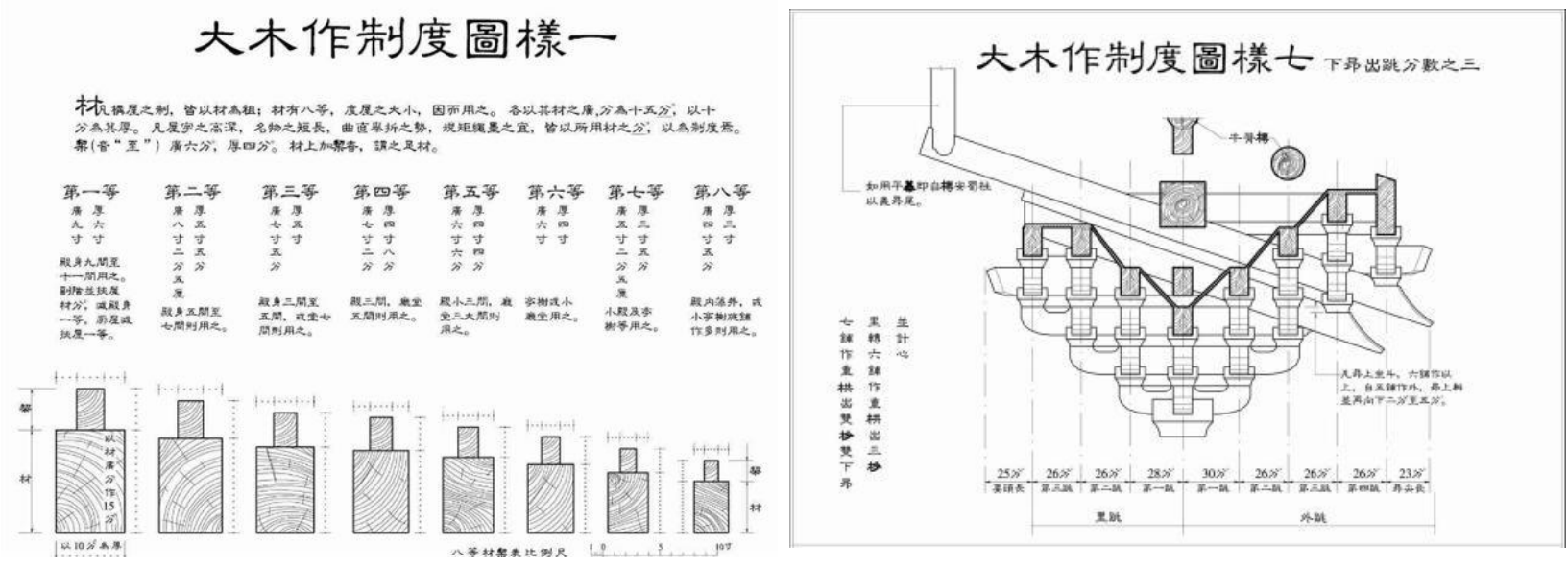

Figure 2. the Corner Bracket Set of Timber Framework

Taking the Corner Bracket Set for example, e.g. Figure 2.

It belongs with the Timber Framework in the 13 METHOD from Ying Zao Fa Shi, which is complex for documentation. Because it contains many rules so that it might has diverse forms depends on diverse inputs, including unit length, level amount, arm type, arm joint form, single or double arm, e.g. Figure 3. Therefore, the algorithm generating would be taking into the practice.

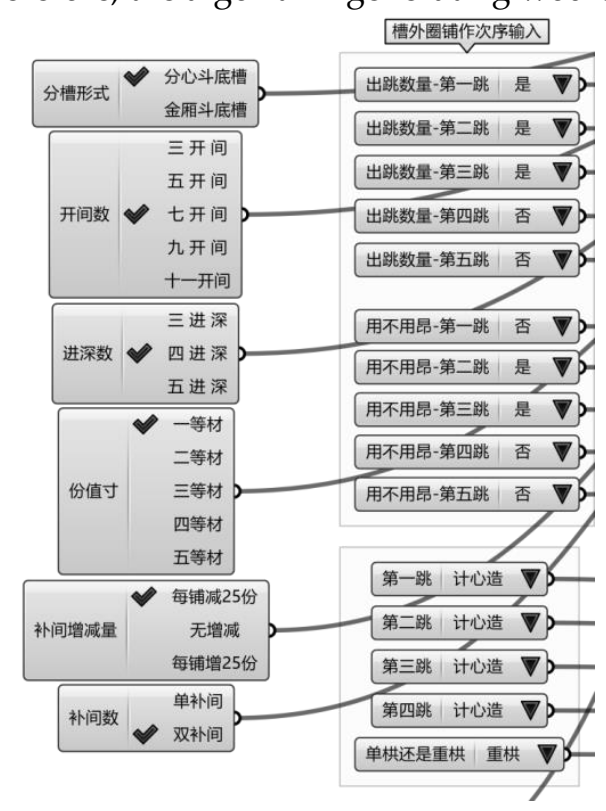

Figure 3. Calculator and Selector
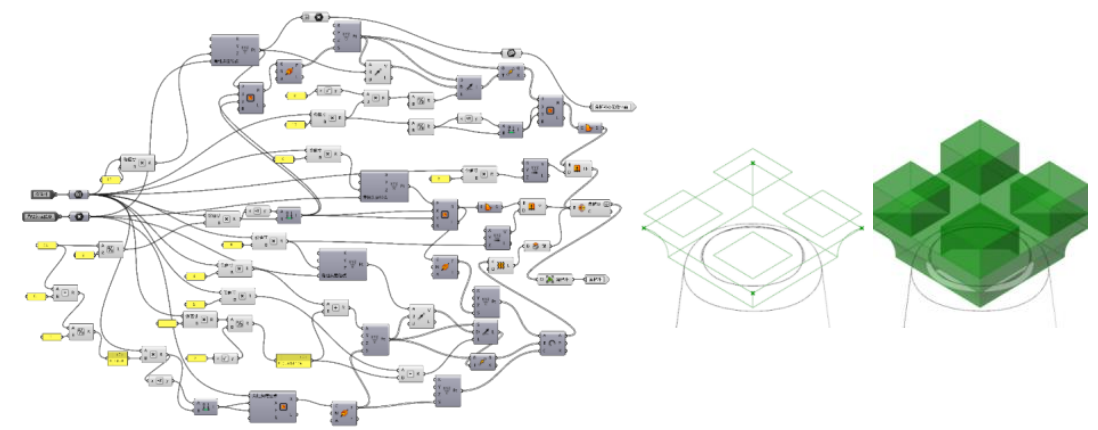

Figure 4. Algorithm battery of Base Generator (left) and real-time result (right) 
Figure 4 shows the algorithm battery of the Base Generator and the real-time result. Then comes the most complex part: Bracket Set Arm and Pad.

If a Bracket Set consists of 1 to 3 levels, then the inner and outer arm of each level both are 30 fen long.

If a Bracket Set consists of 4 or 5 levels ( 5 maximum), then the outer arm of each level remain 30 fen long, while the inner arm of $1^{\text {st }}$ level shortens to 28 fen, and the rest levels shorten to 26 fen, e.g. Figure 5.

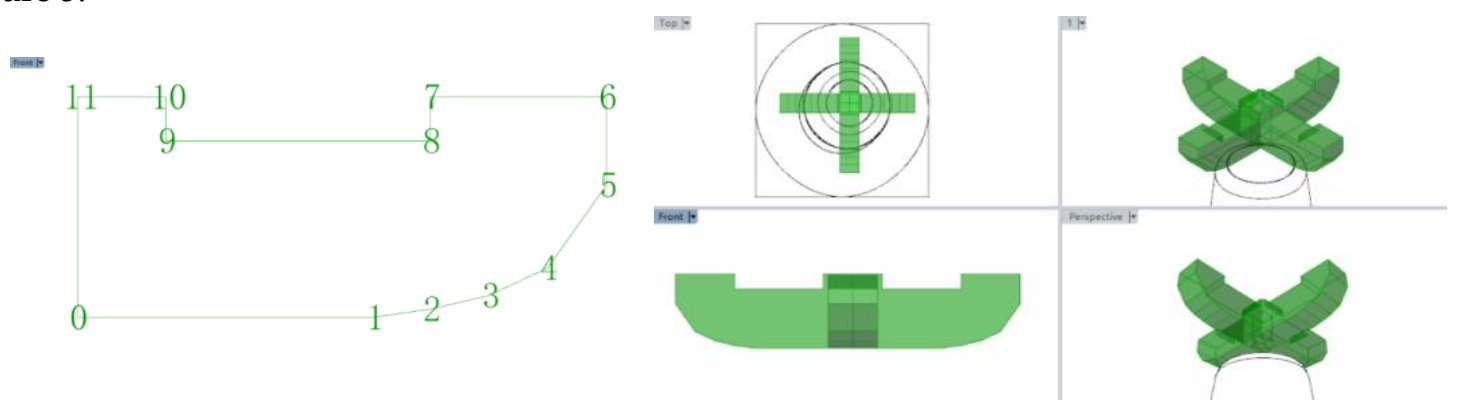

Figure 5. 1st level of Bracket Set with inner arm shortened

Each arm can alternatively be replaced with the cantilever arm, also bringing a dispatch: if using cantilever arm, the $1^{\text {st }}$ cantilever arm should be combined with the normal arm, then rest of them piled together directly.

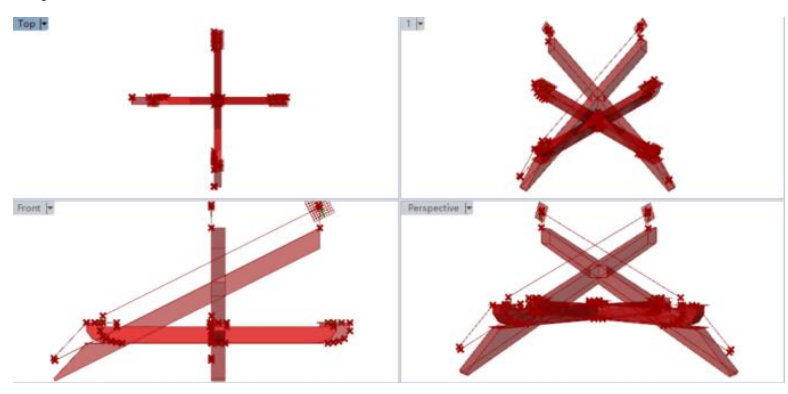

Figure 6. Cantilever Arm combined with Normal Arm

Figure 6 is showing how cantilever arm make the bracket set lower. In this level there are the line frame of cantilever arm, and the solid shape of normal arm it should combined with (if this is the $1^{\text {st }}$ cantilever arm); but there already has a cantilever arm in the previous level, so the solid shape of cantilever arm has been taken; thus making this level lower.

There are 5 arm types: Hua-arm, Nidao-arm, Lower-axial-arm, Upper-axial-arm and Outermost-arm.

Hua-arm jumps out of the Base, sitting cross with the Nidao-arm in each level. No matter how many levels, only one Outermost-arm set on the top of the outer Hua-arm in one Bracket Set. And each Upper-axial-arm should set on the top of Lower-axial-arm (if double arm), or been canceled (if single arm). There are 2 arm tip connecting forms for each level: cruciform joint (touxin zao) and fish-bone joint (jixin zao), making the arm tip various. follows.

Using dispatch battery for Generator output dispatching, and the Boolean input should be as

Lower-axial-arm Generator: 1 for fish-bone joint (jixin zao) and 0 for cruciform joint (touxin zao).

Upper-axial-arm Generator: 1 for double arm and 0 for single arm.

Outermost-arm Generator: Check the equation. Ordinal number of a certain level - Amount of total levels $=0.1$ for true value and output valid data, 0 for faluse value and output null data.

Automatically generating the Corner Bracket Set only by inputting parameters without any manual operation are now achieved. The three instances and corresponding results shown as Figure 7. 
$1^{\text {st }}$ instance: $3^{\text {rd }}$ grade cai, 4 levels including 3 cantilever arms, fish-bone joint (jixin zao) of all arm-tips, double arm.

$2^{\text {nd }}$ instance: $2^{\text {nd }}$ grade cai, 4 levels including 2 cantilever arms, cruciform joint (touxin zao) of arm-tips for 1and 2 levels, fish-bone joint (jixin zao) of arm-tips for 3 and 4 levels, single arm.

$3^{\text {rd }}$ instance: $1^{\text {st }}$ grade cai, 5 levels including 3 cantilever arms, cruciform joint (touxin zao) of arm-tips for 1 and 2 levels, fish-bone joint (jixin zao) of arm-tips for 3 to 5 levels, double arm.

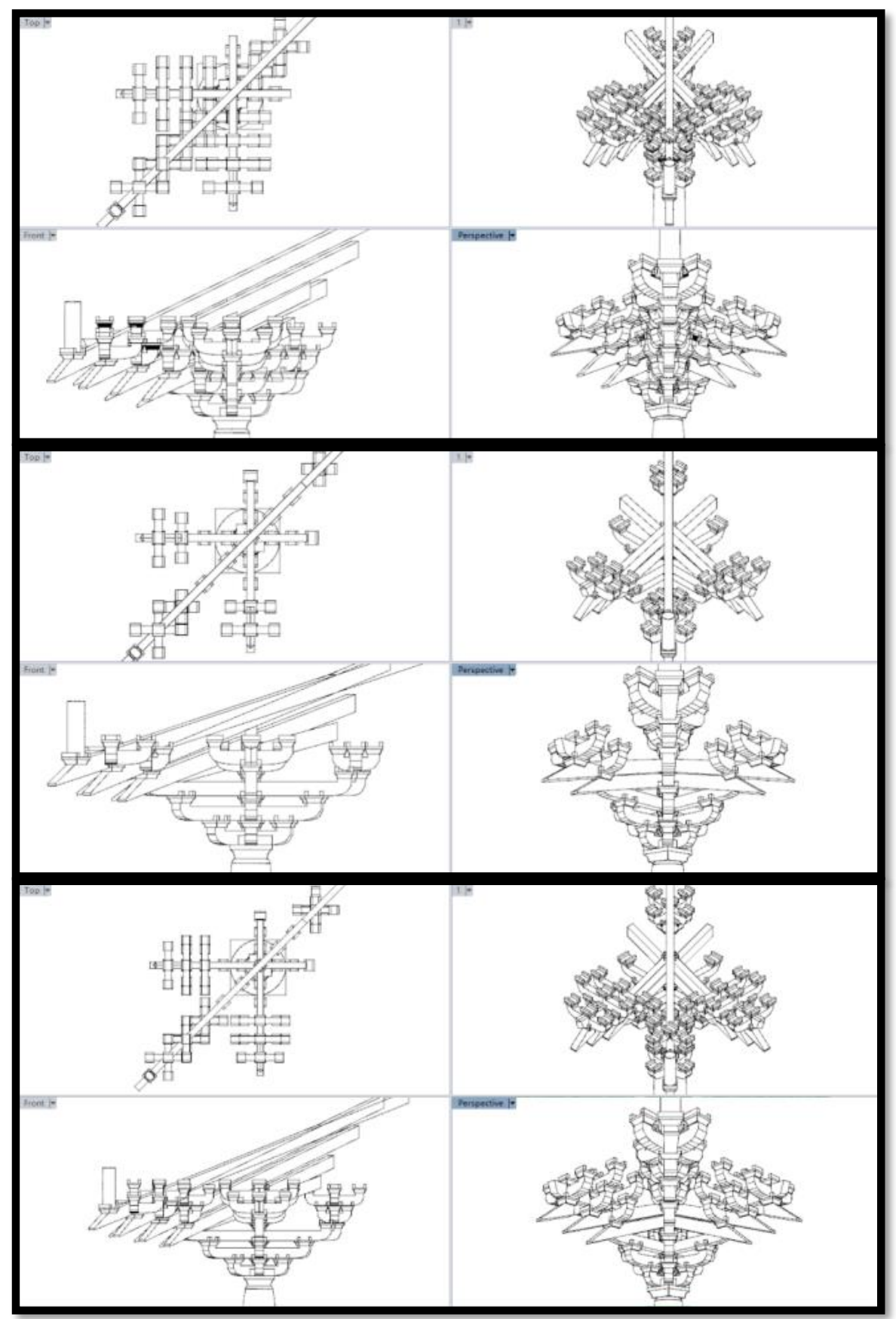

Figure 7. Three instances

This program has already published to the Rhino APP store named Xi[4].

\subsection{Tile Work}

The Tile Work constitute an important part of Chinese ancient architecture, also known as the fifth facade. It belongs to 13 METHOD from Ying Zao Fa Shi, as an independent unit without any 
connections, presenting enormous quantity and rigorous rule, is also complexible for documentation. Therefore, currently the algorithm sorting of standard component would be taking into the practice for reverse engineering, such as the survey and draw of ancient architecture.

Since the hyperboloid exhibited by the tile work is decomposed into every tubular tile and flat tile, the key to rebuild the tile work is rebuilding the hyperboloid by redrawing all the skeleton space curves at key parts referring to point cloud.

First, to acquire a skeleton space curve from point cloud, at least 2 orthogonal projection views is needed. Tracing the top vertex of each eave tile. Generate a polyline curve by vertexs from each view, and then extrude them to get the intersection curve, which would be the skeletion space curve. By all these skeletion curves, the hyperboloid could be rebuilt.

Second, trace the section profile of each component as follows: tubular tile and flat tile, eave tile and drip tile, ridge and tile with animal.

Finally, pick up all the skeleton space curves and section profiles in the Grasshopper, using the auto-sort plugin $W a$ from Rhino App store to generate the whole tile works, and bake them to Rhino.

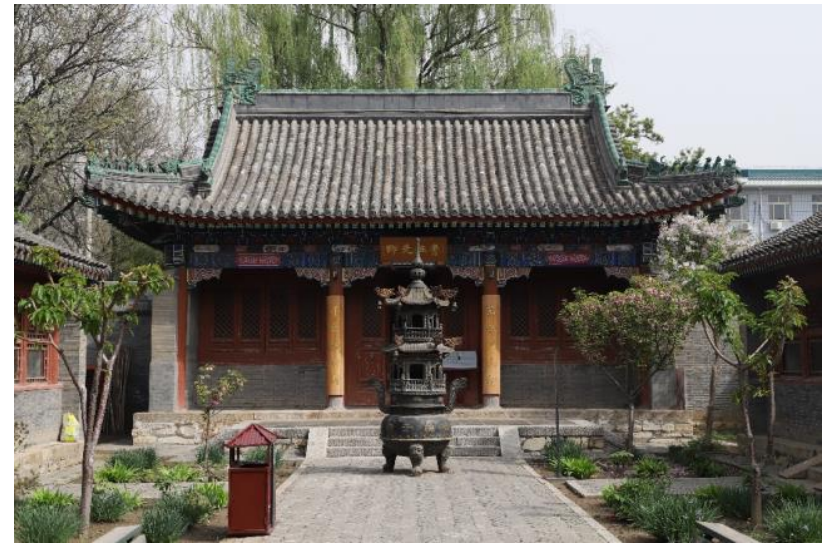

Figure 8. Tianjin Luban Shrine

Taking the Tianjin Luban Shrine for example, e.g. Figure 8 .

Its main hall has a nine-ridge roof. After tracing the top vertex of each eave tile from point cloud, generate the entire skeleton curve of the eaves. Then repeat this workflow to generate the central curve of each ridge, the intersection curve between each top vertex of tubular and the ridge. With the referenced curves and input parameters, the model could be generated, e.g. Figure 9.

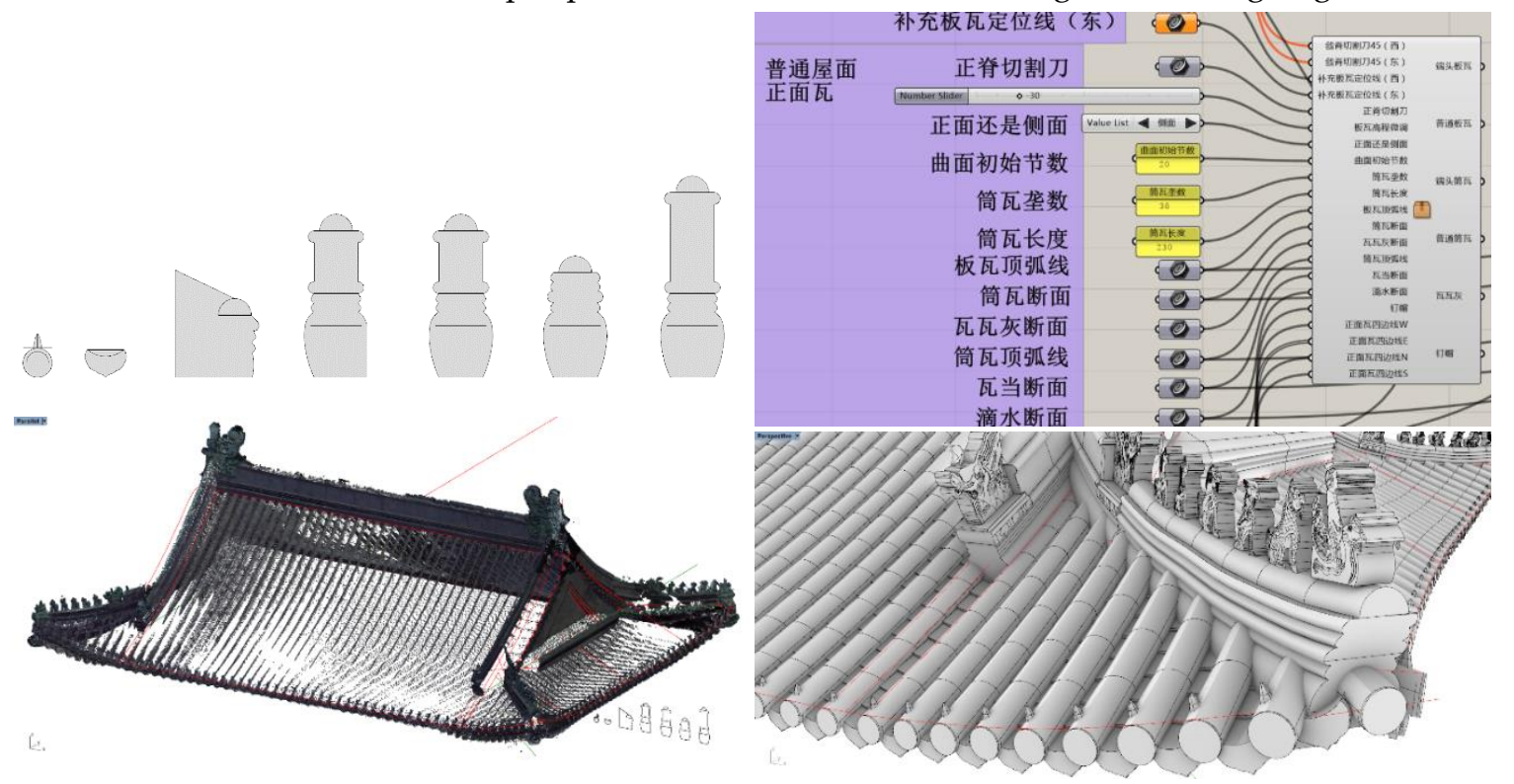

Figure 9. Section profile, skeleton curve, model and input 
The auto-sort plugin $\mathrm{Wa}$ is consist of several algorithm steps as follows[5].

Extract the UV curve from hyperboloid generated by network surface accroding to the skeleton curve, central curve and intersection curve. Then disptch all the $\mathrm{U}$ curves (or V curves depends on the situation) by odd and even check from ordinal numbers. The odd ordinal curves would be the tubular tile crop-row curves and the even ordinal curves would be the flat tile crop-row curves.

Divide each crop-row curve by distance measured from the survey to get the anchor point of each tile.

Evaluate the hyperboloid with the anchor point to get the tangent vector and normal vector of each anchor point.

Move each section profiles to each anchor point, and then rotate separately in $X Y, X Z$ and $Y Z$ plane based on the tangent and normal vector.

Using ruled surface to create each component through each section profile.

\section{Results}

The algorithm modeling realized the forward generating of regular component and the reverse rebuilding of irregular component for the first time, while all the dimension and construction keep associated with the pre-set logic. The corresponding model is created in real-time and would be auto-regenerated according to the inputting parameter or referenced curves without any manual operation.
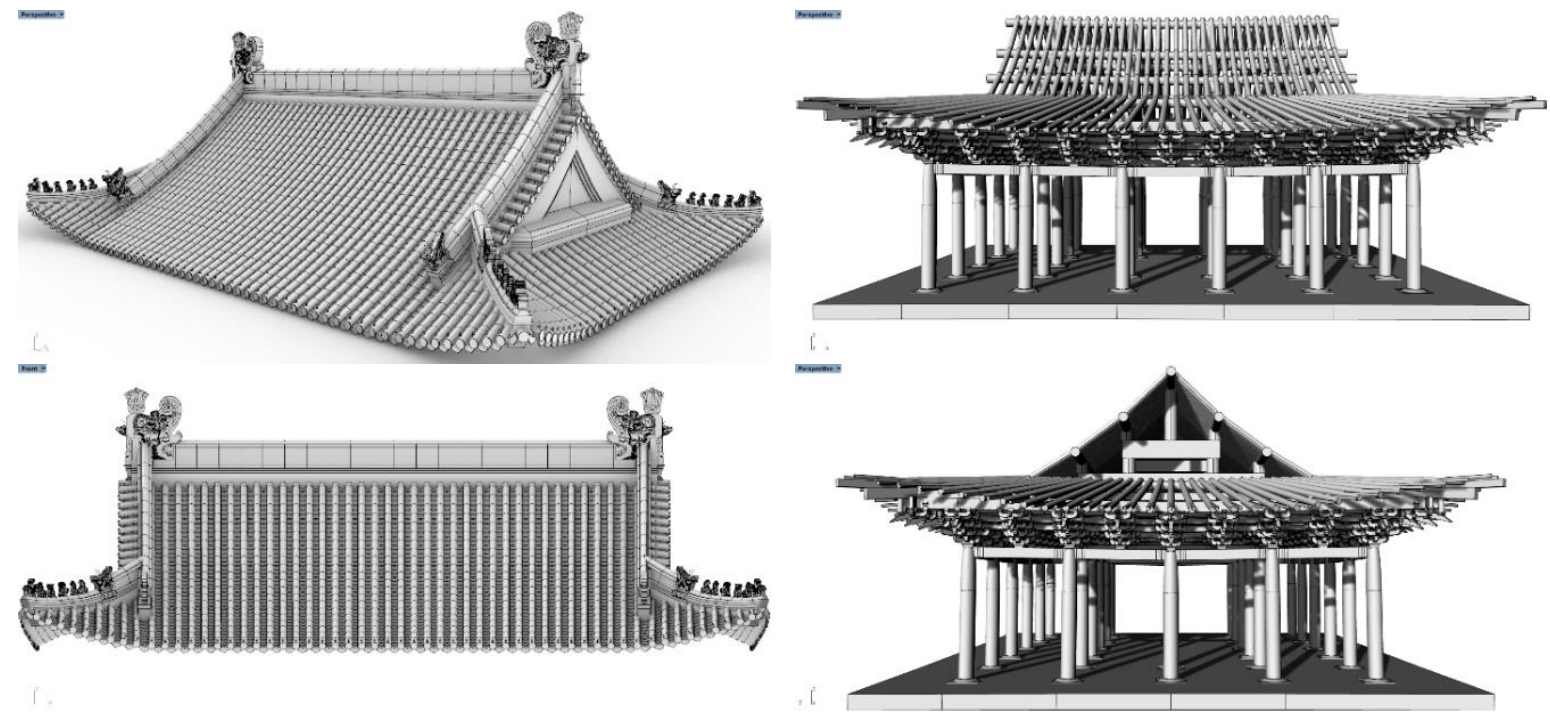

Figure 10. Two plugins of algorithm modeling

The instances of Timber Framework and Tile Work created by the plugin $M u$ and $W a$ proved the velocity and the veracity of the algorithm e.g. Figure 10.

\section{Discussion}

When confronting with the HBIM, it is often been discussed that how to seek the balance between regularization and differentiation. In fact, this is the main issue of HBIM for Built Heritage. The connection rule of each component should be contained in the documentation, but they are not. This is the limit of the current software platforms: parametric component library only expresses the result of the rule, not the rule itself. The Grasshopper now is available in Revit, gives the possibility of algorithm generation, after combining with the point cloud, regularized reconstruction would be realized.

The workflow would be as follows: import the point cloud as reference, express the rules with Grasshopper, adding information in Revit, bake the information model to Revit. 


\section{Patents}

There are three patents under substantial examination.

Author Contributions: Conceptualization, WANG Xi; Data curation, QUE Raner and BAI Chengjun; Formal analysis, BAI Chengjun; Funding acquisition, WU Cong; Investigation, QUE Raner; Methodology, QUE Raner and WANG Xi; Project administration, WU Cong; Resources, QUE Raner; Software, QUE Raner and WANG Xi; Supervision, WU Cong; Validation, BAI Chengjun; Writing - original draft, WANG Xi; Writing - review \& editing, WU Cong. All authors have read and agreed to the published version of the manuscript.

Funding: This research was funded by National Natural Science Foundation of China, grant number 51878440.

Acknowledgments: This paper is partially supported by following institution: the Palace Museum, China; Jiayuguan Academy of Silk Road (the Great Wall) Culture, Gansu; Cultural Heritage Administration of Jizhou District, Tianjin.

Conflicts of Interest: The authors declare no conflict of interest.

\section{References:}

1. 王巍W. Wang; 吴葱C. Wu; 韩涛T. Han 论遗产记录与真实性. 建筑师 2016, 73-76.

2. 陈晓卫X. Chen; 王曦X. Wang 《营造法式》参数化一一殿堂式大木作的算法生形. 华中建筑 2016, $34,41-43$.

3. Jessesn RhinoInside® 简介. In http://tips.cn.rhino3d.com/2019/07/rhinoinside/: 2019.

4. 王曦X. Wang Xi. In https://www.food4rhino.com/app/xi: 2019.

5. 王曦X. Wang Wa. In https://www.food4rhino.com/app/wa: 2019. 